\title{
Bridging the Knowledge Gap

\author{
Examining Potential Limits in Nanomedicine
}

\begin{abstract}
Jaipreet Virdi*
Abstract Nanomedicine has the potential to transform medical therapy and diagnosis. Its technologies predict improved drug delivery systems with sitespecific treatment, precise new surgical techniques that would reduce patient trauma and treatment cause, and even cellular repair that would make agerelated conditions such as Alzheimer's disease a thing of the past. Currently, nanomedicine products are reaching the world market with an annual growth rate of twenty-five percent. However, like any emerging new technology, along with doomsday scenarios of nanoparticles gone amuck, nanomedicine raises serious ethical, moral, and social issues that may potentially limit its technological developments. In this essay, I briefly evaluate some of these issues, and argue that ethical and epistemic issues should be considered prior to research and development. By examining three crucial epistemic challenges in nanomedicine-toxicity, nanodrugs and drug delivery systems, and clinical trials $-I$ argue that by implementing a multi-criterion decision analysis (MCDA) framework as outlined by Linkov, Satterstorm and Corey (2008), these challenges can bridge the knowledge gap between $R \& D$ and the introduction of nanomedicine technologies into the market.
\end{abstract}

\section{Introduction: Defining the World on the Nanoscale}

Viewed as an engine for wealth creation, nanotechnology has become a lucrative investment for major industrial countries that have incorporated fundamental principles of nanoscience into their innovation systems since the late 1990s. These investments, in both government and private sectors, amount to billions of dollars. In his 2000 State of the Union Speech, President Clinton announced the formation of a $\$ 475$ million program for nanotechnology research and development (R\&D), the National Nanotechnology Initiative (NNI). The program's federal funding doubled the next year, and by 2001 annual funding for the NNI exceeded $\$ 500$ million (Freitas Jr. 2005, 2). While the United

\footnotetext{
* Jaipreet Virdi is currently a PhD candidate at the Institute for the History and Philosophy of Science and Technology at the University of Toronto. While she is generally interested in modern issues in the history and philosophy of medicine, her research focuses on early nineteenth-century developments in medicine and biology in England. Her thesis examines the developments of instruments of diagnosis among practitioners specializing in diseases of the ear-particularly John Harrison Curtis (1778-1860)-and how these instruments embodied social and political prejudices against deafness in the nineteenth century.
}

Spontaneous Generations 2:1 (2008). ISSN 1913-0465. University of Toronto 
States leads the field of research and patent productions, worldwide investment in nanotechnology exceeded \$10 billion in 2006. The NNI's current budget for fiscal year 2009 is set at $\$ 1.5$ billion to support basic research, infrastructure development, and technology transfer, validating the claim that nanotechnology is the "next industrial revolution" (National Nanotechnology Initative 2008).

Since Richard P. Feynman's 1959 futuristic vision of a world where microscopic tools built new technologies at the atomic level (Feynman 1959), and K. Eric Drexler's 1989 adoption of Feynman's vision in Engines of Creation, interest in nanotechnology has increased in both academic and public sectors. Nanotechnology is not a single scientific discipline, but a meeting of traditional sciences such as chemistry, physics, biology, and the material sciences. The attraction of nanotechnology is its potential ability to provide a new dimension for technological investigation, manipulation, and application of materials, structures, and devices at the atomic and molecular levels. Nanotechnology typically operates on a scale of roughly 1 to 100 nanometers, where a nanometer is equal to one-billionth of a meter. To understand how small a nanometer is, consider that "[i]f a nanometer were somehow magnified to appear as long as the nose on your face, then a red blood cell would appear the size of the Empire State Building, a human hair would be about two or three miles wide, one of your fingers would span the continental United States, and a normal person would be about as tall as six or seven planet Earths piled atop one another" (Keiper 2003, 19). Properties of every day materials are radically different on the nanoscale, as the large surface-to-volume ratio of elements results in more reactive properties. Aluminum, for example, is ubiquitously used to make harmless soda cans and other goods, and yet in fine powder form it will explode violently in contact with air (Lin and Allhoff 2007, 5). Any term prefixed with "nano" such as nanotechnology, nanoscience, nanomedicine, and the like, focuses its research and application on the nanoscale.

As a result of the limitless possibilities of nanotechnology to ultimately transform many segments of society, nanotechnology products are rapidly reaching the market with an annual growth rate of over 25 percent (Roco 2007, xi). The technology has already made its way into ordinary products such as clothing, sports equipment, and cosmetics (Lin and Allhoff 2007, xv). Engineered nanoparticles may be effective as an environmental agent, providing cleaner air and water, affordable energy, greater computing power, and more. Some of the greatest impacts of nanotechnology are being applied to biology, biotechnology and medicine; this emerging field is known as nanomedicine. While nanomedicine may act as a catalyst for a biomedical revolution, it also raises serious ethical, moral, and social issues. In what follows, I will briefly evaluate some of the issues raised and I will then consider whether nanoethics can readily deal with some of the most pertinent issues facing nanomedicine. 


\section{(Re)considering Nanoethics}

The application of nanotechnology to medicine also foresees promising developments for biomedicine. Nanomedicine is not a single class of medical interventions, but rather an umbrella term encompassing "the process of diagnosing, treating, and preventing disease and traumatic injury, or relieving pain, and of preserving human health, using molecular tools and molecular knowledge of the human body" (Freitas Jr. 2005, 2). With the promise of improved therapy and diagnosis, nanomedicine interacts with fields such as nanoscale surgery, tissue engineering, and the creation of alternative drug delivery systems. In the short term, the benefits of nanomedicine are astounding. New surgical techniques with greater precision could reduce patient trauma and treatment costs. Anti-viral and anti-fungal agents, diabetics, and chronic lung disease managements will be a greater possibility. Medical imaging will allow for a more precise diagnosis, even shifting back to a pre-symptomatic stage to allow for pre-emptive therapeutic measures with less invasive methods of extraction, either in vitro or in vivo. Additionally, new drug delivery systems with pharmacologically-coated nanoparticles will provide site-specific treatment and reduced side effects. Many of the nanomedicine products under development promise to do wonderful things for the current state of medicine, as well as for visionary long-term applications (Thompson 2008). The major longterm application is an extension of gene therapy, with localized treatment-if not cure-of all or some genetic diseases, and nanoparticles could even provide efficient delivery systems for DNA vaccines. A highly desirable feat for nanomedicine would be the development of a cancer vaccine, which might be possible by creating self-assembling biomolecular nanostructures that resemble nanoparticles. These viruses may be coated or use antibodies to destroy cancer cells in any part of the body. Furthermore, advancements in nanomedicine could enable cellular repair, provide cures for age-related conditions such as Alzheimer's disease, and possibly even extend human longevity.

However, despite all the excitement over nanotechnology and nanomedicine, it is likely that many of the long-term predictions overestimate the role and limits of the technology. Prominent scientists, philosophers, policymakers, lawyers, and other scholars in the nanotech field have addressed some of the most relevant current and near-term issues facing nanotechnology, shunning the hyped-up exaggeration and unrealistic expectations of nanotechnology presented to the public by the media and fictional works such as Michael Crichton's Prey (2002). Many of the issues facing nanomedicine overlap issues under consideration in bioethics, particularly issues of autonomy, patientchoice, and justice, in addition to ontological concerns about technologies that are blurring the lines between treatment and change, and the difference between 'natural' and 'artificial' objects. 
Even granting that claims about nanotechnology have often been hyperbolic, and given that viable clinical applications of nanomedicine are years away, it is still important-if not necessary-to address any potential issues for the field while it is still in its infancy. The emerging field of nanoethics, in particular, is concerned with such issues as environmentalism, health and safety, privacy, human enhancements, and humanitarianism. ${ }^{1}$ One effective way to address such issues is by establishing a solid ethical theory for nanomedicine in order to provide a framework for dealing with any short-term or long-term consequences that might arise from the technology. This is particularly important since nanomedicine and nanotechnology are multi-disciplinary fields with different sub-disciplines operating under different codes of ethics. As philosopher Ashley Shew explains, "[a] code of ethics for nanotechnology might be useful as a way to create dialogue about social and ethical implications among the professionals in the field" (Shew 2008, 136). However, do nanotechnology and nanomedicine necessarily require a new code of ethics? Philosopher Christopher Preston points out that we do not enter a new ethical terrain with nanotechnology simply because many of its salient issues can be addressed by environmental ethics. He argues there are only a few cases where we need to seriously consider the implications of emerging nanotechnologies, mainly in cases lacking adequate knowledge of the effects of introducing radically new materials into human and natural environments, or of the abilities of the materials themselves to selfreplicate (Preston 2006). Indeed, the same issues Preston tackles also arise in traditional moral and ethical theories among the works of Thomas Beauchamp, J.F. Childress, and John Rawls. Scholars Mette Ebbsen and Thomas G. Jensen contend that these ethical principles can be extended for ethical assessment in nanomedicine, even though nanomedicine raises ethical concerns that are more complex than those in medicine and biotechnology (Ebbesen and Jensen 2006). There is no need for a new ethical theory for nanomedicine, Ebbsen and Jensen argue, for the ethical principles of bioethics can readily provide an adequate framework to guide nanomedicine.

Nonetheless, no new ethical concerns are raised simply by asking old questions in new contexts (Allhoff 2008, 17). Philosopher Jeroen van den Hoven remarks that "one of the problems with nano-ethics is that it is concerned with problems of future and speculative applications of nano-science. In the first decades of the twenty-first century we still have very few examples of widely used nanotechnology. It is difficult to start a process of reflection on the social and ethical implications of the new technology at the early stages of its

1 For a collection of some of the arguments and concerns, see F. Allhoff and P. Lin's Nanotechnology \& Society: Current and Emerging Ethical Issues (New York: Springer, 2008); F. Allhoff, P. Lin, J. Moor, and J. Weckert's Nanoethics: The Ethical and Social Implications of Nanotechnology (New Jersey: John Wiley \& Sons, Inc., 2007); R.R.H. Coombs and D.W. Robinson's Developments in Nanotechnology, Volume 3: Nanotechnology in Medicine and the Biosciences (Amsterdam: Gordon and Breach Publishers, 1996); Joachim Schummer and Davis Baird's Nanotechnology Challenges: Implications for Philosophy, Ethics, and Society (London: Scientific Publishing Co.Pte.Ltd., 2006). 
development" (van den Hoven 2008, 147). As van den Hoven explains, this problem is an extension of the Collingridge dilemma, where influence over a new technology is only possible at the early stages of the technology's development, even if there is little information about its effects (van den Hoven 2008, 148). This predicament is crucial in nanomedicine, where a few products are already on the market and many more are in development. If nanomedicine, through its promise of improved therapy and diagnosis, has the potential to drastically transform the relationship between the human body and medical knowledge, then we need to consider seriously some of the (long-term) effects of the technology. Take the issue of human enhancement, for example: the potential for disaster in extending human longevity would require new epistemological standards, for "think of all the information that goes into a decision of this complexity. And think of the consequences if we make the wrong choice" (Clement 1998, 405). Not only should ethical considerations take place before $R \& D$, but we need to understand and clarify any epistemic issues in nanomedicine to account for knowledge gaps within the development and application of the technologies.

The European Group of Ethics in Science and New Technologies 2007 report stated that "the uncertainties and knowledge gaps associated with new nanotechnology-based diagnostics, therapies, and preventive measures should be identified. These uncertainties need to be characterized and measures have to be developed in order to reduce them as far as possible" (European Group on Ethics 2007).The uncertainties especially need to be evaluated in light of the possibility that the hype surrounding nanomedicine research will obscure ethical issues and larger implications of R\&D. As leading researchers in nanomedicine Raj Bawa and Summer Johnson explain, "[o]nce the product is on the market, it is difficult to put the genie back in the bottle, particularly if market forces dictate otherwise" (Bawa and Johnson 2008, 212).It is critical that research risks and any long-term ill-effects be carefully evaluated. But how do we evaluate these risks while balancing ethical, social, and moral considerations?

Bawa and Johnson raise an additional point: "[b]ecause it is difficult to exactly predict technology trends or innovations in nanomedicine, it is impractical for ethicists to envision or address all possible scenarios or issues that might arise of nanomedicine in the future" (Bawa and Johnson 2007). Considering that nanomedicine is not a single class of medical interventions readily available to analyze from an ethical perspective, advances in nanomedicine and medical care should not arrive at the expense of safety. Further, since many of the issues in nanomedicine, such as the issue of toxicity, are empirical issues, not moral or conceptual ones, they should be addressed early on along with any ethical issues. Through the remainder of the paper, I will examine three closely-related epistemological challenges that are currently being debated in nanoethics and may potentially limit public acceptance of 
nanomedicine technologies, issues that demonstrate the importance of carefully addressing epistemological questions. The three challenges are: toxicity, nanodrugs and drug delivery systems, and clinical trials.

\section{Toxicity}

The most significant current and near-term concern of nanomedicine is whether or not nanoparticles or nanomaterials pose any inherent threat to human health or to ecological systems, especially since there is a lack of solid experimental data on unintended and adverse effects. The same properties that make nanomaterials desirable may also increase their toxicity; in particular, there is an increasing concern among scientists and nanoethicists about how nanoparticles might affect or interfere with the biochemical pathways and processes of the human body" (Garber, 2007) Additionally, the issue of safety over the handling and disposal of nanoparticles has received attention from the International Risk Governance Council (White Paper Nanotechnology risk governance, June 2006) and the European Group on Ethics (January 2007), and the European Scientific Committee on Emerging and Newly Identified Health Risks (SCENIHR) acknowledged that major gaps in knowledge need to be filled in relation to improved risk assessment for products of nanotechnology and nanomedicine. The SCENHIR report (September 2005) further elaborated that

in spite of a rapidly increasing number of scientific publications dealing with nanoscience and nanotechnology, there is insufficient knowledge and data concerning nanoparticles characterisation, their detection and measurement, the fate (and especially the persistence) of nanoparticles in humans and in the environment, and all aspects of toxicology and environmental toxicology related to nanoparticles, to allow for satisfactory risk assessments for humans and ecosystems to be performed (SCENHIR 2005).

The report takes into account that no general statements on hazards or toxicities of nanomaterials to humans can be made for risk analysis, for the potential ill-effects of nanoparticles can be derived from data of bulk materials. Rather, studies should operate on a case by case basis, carefully evaluating the toxicological effects of the nanomaterial used in a particular case. The Australian Safety and Compensation Council (ASCC) has also found that "the health risks of nanostructures cannot be predicted a priori from their bulk equivalent and that animal studies in this area, although limited, raise serious concerns" (Faunce 2007, 189).

Central to the health risk concerns of toxicity is the ability of the human body's defence system to react against nanoparticles. Studies on animal and human cells (both in vitro and in vivo) have begun to gather significant data on the toxicity of nanoparticles. These studies demonstrate that nanomaterials can move from the exposure site to other parts of the body, and inhaled nanoparticles can enter the bloodstream and hitch a ride to various organs. 
Nanoparticles can even be deposited in the respiratory tract after inhalation, or penetrate the skin, travel to the lymph nodes, and cross the blood-brain barrier (Resnik 2006). Despite concerns of health and safety risks associated with the presence of nanoparticles in the body, there is a significant lack of quantitative analysis and empirical data on risk assessments and nanotoxiology. Much of this is due to scientific uncertainty over long-term medical reactions, and due to demonstrations that immune system failures due to the presence of nanoparticles distort empirical data for analyzing whether the nanoparticles are toxic. Other various reports also stress that further data on the risk assessments of toxicity and human health is essential before any nanomedicine products hit the market, since a greater concern is raised from the spread of free nanoparticles in the environment rather than individual exposure.

Research has only recently begun analyzing adverse health reactions to free nanoparticles and the long-term safety of engineered nanoparticles (ENPs). Early experiments on laboratory animals have demonstrated a high level of toxic reaction to ENPs, and short-term exposures on animals have produced dosedependent inflammatory responses and pulmonary fibrosis (Faunce 2007, 190). Bioethicist Thomas A. Faunce argues that as chronic in vivo studies detailing precise experimental data on toxicological effects of nanoparticles are yet to be published, we still need to consider the tendency of some ENPs to accumulate in mitochondria and inhibit function, while other ENPs might enable unstable biological settings within an organism and release toxic elementary metals (Faunce 2007, 190). In addition, the leading expert in nanotoxicology, Günter Oberdörser at the University of Rochester, also points out that a nanoparticle's behaviour is often unpredictable: they may behave differently in vivo than in vitro, may disintegrate into even smaller particles that are even more toxic, or cluster together in dangerous groups (Oberdörser et al 2007).

If toxicological concerns are to be addressed prior to $R \& D$, how do we address these concerns in light of the lack of solid empirical data? Serious empirical studies on toxicity are essential for bridging the knowledge gap between research, and for the introduction of nanomedicine products into the market. This is a difficult endeavour, since risk assessment in nanomedicine differs from traditional frameworks applied in other biosciences. While nanomaterials can be subjected to assessments of hazard identification, it is difficult to analyze data related to toxicity, risk, and exposure assessments. One key factor for this problem is that nanotoxicology requires specific knowledge about the metabolism and distribution of nanoparticles in the body, and even though a variety of techniques are available, current toxicological screening methods may not have the resolution necessary to provide useful information at such small scales. Additionally, questions of whether particular metabolized nanoparticles stay localized or re-enter the circulatory system are difficult to answer empirically at the present stage of nanomedicine (Linkov, Statterstsorm 
and Corey 2008, 168). The European Group on Ethics contends that "only a few specific nanoparticles have been investigated in a limited number of test systems, and extrapolation of this data to other materials is not possible" (European Group on Ethics 2007). The Group concurs that these few specific examples all indicate that known and widely accepted toxicological methods are not sufficient to detect possible damaging effects of nanoparticles.

If risk assessment for toxicological concerns in nanomedicine is to proceed in bridging the knowledge gap, a sufficient epistemological model is needed to balance the risks, benefits, and scientific uncertainty of nanoparticles. Understanding nanomedicine toxicity requires a complex set of data, one which consolidates information about optimal nanomaterials and nanoparticles in individual situations in order to understand their long-term toxic effects. To date, an adequate model has been supplied by Igor Linkov, F. Kyle Satterstorm and Lisa M. Corey, who merge an innovative combination of nanotoxicology, risk assessment models, and analytical tools developed in the field of multi-criterion decision analysis (MCDA) for evaluating long-term effects of toxicity from nanomedicine products. The MCDA method is used within the U.S. Department of Defence and the Environmental Protection Agency, and as a tool for integrating heterogeneous information for regulating decision-making and analyzing negative consequences that may arise, and the method can readily be applied for evaluating nanomedicine concerns about toxicology. As Linkov, Satterstorm and Corey elaborate, the "MCDA framework links heterogeneous information on causes, effects, and risks for different nanomaterials with decisive criteria and [weights-of-evidence] elicited from decision makers, allowing visualization and quantification of the trade-offs involved in the decision-making process" (Linkov, Statterstsorm and Corey 2008, 170). This framework will allow efficient decision-making processes on appropriate nanomaterials for medical applications while balancing considerations of medical factors and side effects with associated uncertainties related to toxicity. A MCDA framework will focus on two approaches: (1) generating alternative nanomaterials and treatment options, success criteria, and value judgements, in order to generate and define particular choices and preferences; and (2) ranking the alternatives by applying value weights in order to judge criteria levels generated by toxicity models, experimental data, or expert judgement (Linkov, Statterstsorm and Corey 2008, 169).

As an analytical tool, the MCDA framework will be applied to generate and map technical and empirical data as well as individual assessments into organized structures to be linked with other technical tools from risk analysis, modeling, monitoring, and cost estimations (Linkov, Statterstsorm and Corey 2008, 170). In particular, the authors emphasize an adaptive management structure that will center as a means for incorporating changing decision priorities or new knowledge from toxicology screening and studies. Since stakeholders, scientists, doctors, managers, and patients all have their own way of viewing the problem of toxicity and offering various solutions, the MCDA 
framework will allow an interactive engagement of decisions that will comprehensively structure a process for selecting alternatives in a given situation. As the authors contend, this "structured process would be of great benefit to decision making in nanotechnology management, where there is currently no structured approach for making justifiable and transparent decisions with explicit trade-offs between social and technical factors" (Linkov, Statterstsorm and Corey 2008, 171). They also argue that side-effects regarding the use of nanoparticles and nanomaterials need to be considered and evaluated during the engineering process, and in order to combat any scientific uncertainty, "decision makers need an understanding of product life cycle and the ability to communicate effectively with personnel, stakeholders, and regulators" (Linkov, Statterstsorm and Corey 2008, 167).

The MCDA model provides a solid empirical and analytical approach for evaluating toxicological data and dealing with long-term uncertainties and risks in the use of nanoparticles by sustaining a commitment to scientific justification without abandoning a commitment to social and medical assessments. In doing so, the model justifiably provides decision-making criteria for evaluating and applying a proper assessment of the risk and safety of nanomedicine products, especially in a lack of solid empirical data on the toxicity of nanoparticles and nanomaterials. The model can also be readily applied in assessing risk vs. benefit or drug delivery systems, and the regulations of clinical trials using nanomedicine technologies. A proper assessment of the risk and safety of nanomedicine is required in order to ensure the safety of participants in any clinical trials of nanomedical products, and no products should enter the market without proper risk assessment. Furthermore, the challenge of toxicity is closely related to the effects of nano-drug delivery systems, which I shall discuss in the next section.

\section{(Nano-)Drug Delivery Systems}

Drug delivery systems are the main commercial application for nanomedicine as pharmaceutical companies recognize the growing impact of this technology on the market. Drug delivery systems account for $78 \%$ of global sales in nanomedicine and $58 \%$ patent filings worldwide, and according to the NanoMarkets Report they will generate over \$1.7 billion U.S. dollars in 2009 and over $\$ 4.8$ billion by 2012 (Bawa and Johnson 2008, 208). The most basic drug delivery systems enhance the effectiveness of pharmaceutical drugs by targeting certain types of cells, speeding up delivery time, and preventing digestive enzymes from breaking down the medication (Miller 2003, 7). With the "potential to address unmet needs and personalized medicine" (Bawa and Johnson 2008, 208), drug delivery systems based on nanotechnology have the potential to drastically improve medical therapeutics and diagnosis. These systems use nanodrugs, "a heterogeneous groups of drugs that generally offer unique properties because of their nanoscale dimensions" (Bawa and Johnson 
2008, 214), and vary in shape, size, and chemical composition. Some of these nanodrugs are actually nanoparticles coated with active pharmaceuticals that provide site-specific treatment. This type of technology is highly favourable with the potential to travel through the blood and across the blood-brain barrier to include specific reagents (such as antibodies) that are able to bind to specific targets. The benefits of these systems are highly desirable: fewer side effects, improved drug efficiency, and treatment of diseases that are presently untreatable.

Developments in nanomedicine and pharmaceutical R\&D have begun to incorporate valuable medical diagnosis with drug delivery systems, building a solid path towards clinical therapeutics with nanodrugs. These drugs can also be attached to nano-carriers, which can be engineered to be activated by environmental changes such as body $\mathrm{pH}$, chemical stimuli, or an external heat source (Ebbesen and Johnson 2006, 7). In an experimental treatment in Berlin, amiosaline-coated nanoparticles were injected into a cancerous tumor and subsequently heated with a magnetic field applicator. Due to their unique properties, the coated nanoparticles formed stable deposits within the tumor, which in turn rapidly absorbed the coated nanoparticles and was eventually destroyed by heat after repeated treatments (Wagner, Dullaart, Bock and Zweck 2006, 1214). A similar drug delivery method uses nanoshells-gold-coated silica embedded in a coat of tumor-targeting hydrogel polymer-which are injected into the body in order to accumulate near tumor cells and are then heated with an infrared laser, releasing drugs at a specific site (Freitas Jr. 2005, 5). This method will not only improve the treatment of cancerous tumors, but can be modified for treatment for other diseases, such as diabetes. Additionally, fullerene-based pharmaceuticals have also demonstrated success in serving as antiviral agents, antibacterial agents, photodynamic antitumor and anticancer therapies, antioxidants and antiapoptosis agents for amyotrophic lateral sclerosis and Parkinson's disease (Freitas Jr. 2005, 5).

However, despite the astounding benefits of nanodrugs and nanomedicine drug delivery systems, critical ethical and epistemic issues are raised. Despite the hype of a new technology and the possibility of revolutionary breakthroughs in medicine, are the nanomedicine drugs any more effective and safe for humans than conventional drugs? Much like the issue of toxicity, there is a significant lack of empirical data supporting the claim that these nanodevices and nanodrugs would not trigger a severe immune response, cause toxicity, or penetrate the cell nuclei and trigger an undesirable genetic mutation or gene response. There are no convincing studies analyzing the effects of heat (even through infrared laser) for site-specific treatment, nor the possibility of heat damage to healthy cells. Moreover, "how do we check that, because of their greater capacity to pass through biological systems (for instance, crossing the blood-brain barrier and penetrating the brain), that nanodevices designed for drug delivery will not induce negative side effects for the patients?" (European Group on Ethics 2007). What implications will rise from the presence of different 
nanomaterials and nanodevices carrying the nanodrugs through the body? Furthermore, how will these nanomaterials and nanodevices be disposed of once the treatment is over?

These issues require full risk assessment and quantitative empirical studies before such treatments are fully implemented in clinical trials. While the MCDA model may adequately provide an approach for dealing with communication and information through multi-dimensional levels, it cannot adequately account for any long-term shortfalls of nanodrugs and drug delivery systems. For one thing, much like other conventional pharmaceutical drugs, nanodrugs and drug delivery systems raise epistemological issues related to patient treatment: how does knowledge gained from clinical trials using nanodrugs serve as a guideline for what constitutes the best treatment for the patient? How does a physician decide to include a patient in nanodrug clinical trials? It is not even entirely clear how knowledge gained from research evidence of nanodrugs answers what is the best treatment for the patient, or how to homogenize risks across different patients. These issues are similarly raised by proponents of evidence-based medicine who recognize that there appears to be a knowledge gap between accumulating research knowledge and applying treatment to a patient based on the research knowledge. There is no solid justification of the belief that a particular (nano)drug would be effective for this patient simply based on an empirical demonstration of superiority of this particular (nano)drug, for that justification cannot predict possible long-term consequences that may arise within the patient. Given that in $2000,15 \%$ of all hospital admissions were due to adverse drug effects, resulting in 100,000 deaths (Lazarou, Pomeranz and Corey 1998), we need to seriously evaluate the potential disastrous consequences that may arise from the use of nanodrugs and drug delivery systems without proper empirical studies.

In light of these epistemic gaps, scientists, researchers, and physicians have a moral responsibility to address the ethical principle that patients should not be exposed to treatment or research without their free and informed consent. This might be difficult considering the lack of sufficient empirical data on long-term risks and consequences that may arise with nanodrugs and drug delivery systems. While notifying the research subjects of these risks may may make it difficult to recruit them for clinical trials, withholding this information for the purposes of research will seriously violate ethical and moral principles. With such a dilemma, how should researchers deal with balancing their lack of knowledge about risks with their duty to inform the patient or research subject? Additionally, the complexity of nanotechnologies may cause problems with comprehension and understanding for those wishing to participate in such trials (Bawa and Johnson 2008, 216). Here, the MCDA model can be applied for transmitting information and opening communication with patients. Even if adequate empirical studies have not been conducted, patients should be notified 
of the existence of potential and unpredictable long-term risks. Patients and research subjects should not be treated as "passive objects that have evidence applied to them after information has been extracted from them" (Upshur and Tracy 2004, 198), but rather encouraged to be active participants in a therapeutic relationship with the physicians and researchers. Moreover, as Bawa and Johnson emphasize, there is a moral responsibility on the part of R\&D scientists to allow for long-term follow-up with patients receiving a nanomedicine, even though long-term follow-up assessments (e.g. postmarketing surveillance or Phase IV studies) are generally poorly practiced due to insufficient legal regulations (Bawa and Johnson 2008, 216).

Additionally, economic considerations of nanodrugs and drug delivery systems need to be evaluated. Due to the exaggerated hype of new, innovative technologies, and high expectations from the public and stakeholders for a revolutionary new medicine, researchers and scientists are faced with extreme pressure to deliver nanomedical technologies and drugs that will live up to the hype without properly assessing ethical and empirical considerations. These public pressures translate to corporate pressures, particularly in the pharmaceutical drug industry, where the driving force of innovation is wealth generation rather than knowledge generation. As Bawa and Johnson explain,

In fact, the cost of developing and launching a new drug to the market, although widely variable, may be upwards of 800 million US dollars. Typically, the drug appears on the market some 10 to 15 years after discovery. Furthermore, for every 8,000 compounds screened for potential drug development, only one makes it to final clinical use, and only one out of five lead compounds make it to final clinical use... While the costs of drug R\&D continues to rise, only $30 \%$ of drugs are able to recover their R\&D costs (Bawa and Johnson 2008, 211).

With such high investment costs in the production of nanomedicine technologies, it is crucial that epistemic and ethical evaluations for any dangerous consequences are not ignored; doing so would create a similar extensive public outrage as against genetically-modified foods, or tobacco companies. One approach for avoiding such public backlash is to enforce the publication of all research results-whether negative or positive. Publications must be contractually obliged on the part of the researchers on any nanodrug and drug delivery systems, especially for clinical trials using human subjects, in order to establish secure communication between researchers and stakeholders, and to secure public trust in the technology. In the next section, I will look at the role of communication and publication of results of nanodrugs and drug delivery systems within clinical trials. 


\section{Clinical Trials}

Several writers have voiced their concern with the U.S. Food and Drug Administration's (FDA) inadequate preparation of new application procedures for nanomedicine technology, and the regulations for approval of clinical trials governed by the FDA regulatory agency. John Miller, for instance, writes that the FDA's failure "to effectively regulate nanomedical products could be disastrous for public health, the emerging nanotechnology industry, and the FDA" (Miller 2003,2 ). While examining some of the potential issues facing the FDA in regards to regulating nanotechnology, Miller contends the FDA will face great difficulty in terms of classification and maintaining strict regulations. Regardless of the difficulty in regulations, as of 2006, the FDA has recognized the growing applications of nanomedicine, particularly the benefits from nanodrugs and drug delivery systems, and evaluated several nanomedicine products for clinical trials.

The regulation of drugs under the FDA amendments requires a manufacturer to first file an Investigational New Drug (IND) application for approval for research using human subjects. The manufacturer must identify the target for the new drug and conduct small-scale preclinical studies demonstrating that the drug is safe to use in order to regulate risk vs. benefit assessments. Once the projected benefits outweigh the projected risks, the FDA determines whether the drug can be approved. Upon completion of preclinical research, the manufacturer then applies for a New Drug Application (NDA) to study the drug through three phases of human clinical trials. Once these clinical trials are completed, the FDA then decides the final approval for the manufacturer's marketing of the drug.

As early as 2001, nanodrugs and nanomedicine devices have received FDA approval for clinical trials. Copernicus Therapeutics has tested nanoparticlesbased gene therapy in phase I clinical trials (Haberzettl 2002, 12). The first FDA approval of a nanodrug was in 2005, with collaborations between pharmaceutical companies Merck, Wyeth, and Abbot, on the development of a nanoparticulate drug using technology developed by Elan Drug Delivery. These drugs, commercially marketed respectively as Emend, Rapamue, and TriCor, transformed guidelines for patent terms and exclusivities (Till, Simkin and Maebius 2005). The latest approval for clinical trials as of printing was announced on April 22, 2008, with the approval for Calando Pharmaceuticals' (a subsidiary of Arrowhead Research Corporation) Investigational New Drug application for its anticancer compound, CALAA-01. The drug candidate is a targeted nanoparticle, and the FDA approval allows for the initiation of phase I clinical trials, making CALAA-01 the first clinical study using a targeted, systemic delivery of siRNA in an oncology setting (Nanotechnology News April 22, 2008). Currently, diagnostic tests and drug delivery systems are the main types of nanomedicine products in clinical trials. 
Several problems are raised with FDA-approved clinical trials for nanomedicine technology. The first major concern is raised by bioethicist David Resnik, who argues that any toxic accumulation in tissues or genetic mutations resulting from the use of nanodrugs or the implementation of any nanoparticles, nanodevices, or nanomaterials would not appear at the normal length of a clinical trial, or even during the FDA approval process. While it takes roughly seven years for a company to bring a new drug through the entire FDA approval processes, and an additional few years for post-marketing studies, significant long-term risks may not surface for fifteen or more years (Resnik 2006). Resnik further argues that even animal studies generally last only between 28 to 90 days and rarely investigate any possible long-term effects that may arise in human subjects. Once preclinical testing on animals approve the safe use of a new nanomedicine product, the product is then evaluated in a phase I clinical trial for human subjects to determine the safety of the product for human use and any side-effects that may arise.

Resnik argues that the small number of human test subjects in phase I clinical trials (25-100) cannot adequately detect rare side effects that may affect susceptible subpopulations (Resnik 2006). Additionally, a drug may enter the market after clinical trials have been done on fewer than a thousand subjects, when according to new regulations set by the FDA's post-marketing surveillance, at least three thousand subjects are needed to demonstrate a ninety-five percent chance of detecting serious side effects (Resnik 2006). One possible reason for the small number of human test subjects may be the difficulty of recruiting patients for clinical trials, which may be partly due to the relatively new state of nanomedicine and the lack of public trust in it. In an article in New Scientist, Michael Brooks nicely captures the weak relationship between public trust in nanoscience and the amount of information available to the public: "It still isn't clear whether anyone actually wants to be a nanomedicine guinea pig. It's all very well to dream up and develop cell repair machines, but what if the prospect of rampaging nanorobots or unexpected immune reactions means that no one is prepared to have the technology implanted?" (Brooks 2001, 33).

Additionally, as previously mentioned, the issue of public trust in nanoscience and the complexity of nanomedicine might make informed consent for human subject research increasingly complicated. Considering that not all long-term risks might be readily apparent at the start of a clinical trial, researchers have a duty to fully disclose all possible unpredictable risks that may arise during the trials (Bawa and Johnson 2008, 212). Physicians in particular need to be sensitive to the issue of risk, since by "not openly discussing risks and benefits together, trust is difficult to re-build. Low levels of trust make technologies inherently unstable from a social perspective, and often lead to overreaction" (Metha 2003, 17). Furthermore, even preclinical animal studies are not empirically sound vehicles to provide insight for risk assessment in humans, since animals and humans may react differently to the same nanomedicine products. In a phase I experiment in the United Kingdom, six 
volunteers became critically ill after receiving a monoclonal antibody after preclinical animal trials showed no signs of toxicity, even though the animals had received up to five hundred times the dose used in the human subjects (Resnik 2006). Further toxicology studies are required to quantify the degrees of risks to human subjects and to properly assess risks facing human subjects, even if animal studies have shown tremendous promise in the nanodrug or nanomedicine technology. This is especially essential if the nanomedicine technology is to pass through clinical trials for patent production and commercialization.

Proper clinical protocol and full disclosure of risks in human subject research involving nanomedicine technologies is crucial for avoiding another Jesse Gelsinger scenario. Gelsinger is the first publically acknowledged death directly resulting from gene-therapy, and he serves as the human face of the need for proper clinical trials and full disclosure to patients. 18-year old Gelsinger suffered from ornithine transcarbamylase (OTC) deficiency, a rare metabolic disorder caused by a genetic mutation, which he controlled with a low-protein diet and thirty-two pills a day. Severe OTC deficiency typically affects newborns, who slip into coma within seventy-two hours of birth. Half die within the first month, and many survivors do not make it past their fifth year. The University of Pennsylvania was conducting experimental gene-therapy trials aimed at testing the safety of a treatment for babies with a fatal form of the disorder. In fall of 1999, Gelsinger joined the clinical trial at Penn, though he knew the study was aimed at testing newborns. On Monday September 13, he was injected with adenoviruses (which had been used for one-quarter of all gene therapy trials) carrying an infusion of corrective genes, which had previously been tested on mice, monkeys, baboons, and one human patient. Researchers were aware that the drug had side effects, but in the past they had been limited to flu-like symptoms and mild liver inflammation. On Friday September 17, Gelsinger suffered a massive immune response triggered by the viral vector, eventually dying from multiple organ failure and brain death (Stolberg 1999).

Like nanomedicine and nanotechnology at present, gene therapy was a promising idea that was expected to drastically reshape the medical landscape. Biotechnology companies invested billions into R\&D for finding genetic cures for rare hereditary disorders, and "big-profit illnesses like cancer, heart disease and AIDS" (Stolberg 1999). The pressures to deliver favourable positive results were felt by the researchers at Penn, and this pressure might have significantly undermined their adherence to proper clinical protocols. After Gelsinger's death, all trials using methods similar to those of the Penn study were suspended, and preliminary results by Penn were released under pressure from a public inquiry headed by the Recombinant DNA Advisory Committee (RAC). As Sheryl Stolberg reported, similar research headed by the Schering-Plough Corporation showed that patients experienced serious side effects, including changes in liver function 
and blood-cell counts, mental confusion, and nausea, and two even experienced minor strokes (Stolberg 1999). Further investigation by the FDA concluded that Penn should have not enrolled Gelsinger as a substitute for another volunteer who dropped out, and the university failed to report severe side effects experienced by two other patients. Paul Gelsinger, Jesse's father, has publicly declared the failure of the FDA for disclosing vital information about risks, including the deaths of monkeys receiving similar treatments, stating in a letter to the editor of The Daily Pennsylvanian: "The harshest truth came in December when I discovered that the efficacy that had been represented to me, and thus to Jesse, in July 1999 had never existed and that at least one monkey had become very ill using the same altered virus that Jesse received" (October 2, 2000). Paul Gelsinger is now an advocate of proper disclosures of informed consent both to voluntary subjects for clinical trials, and to the families of the subjects. He has tirelessly argued, in the name of his son, that industry secrets, patent confidentialities, and financial alliances should not trump patient rights.

The lesson from Jesse Gelsinger demonstrates the need for proper communication channels and full disclosure to patients enrolling in clinical trials. For nanomedicine this may be troublesome, for it is difficult to predict long-term risks that might surface after the trials have been concluded. However, the hype and hope should not exceed realistic expectations, and transferring research outcomes from the laboratory to commercialization needs to be carefully governed. Without proper risk assessment, the consequences will be disastrous. Implementing the MCDA framework will make room for an additional shift from risk assessment to risk management, even after post-marketing surveillance, and thus ensure that any dangerous consequences will not be ignored for economic gains. This is especially necessary if regulators, researchers, and industry experts are to design a new clinical protocol to deal with unpredictable results from nanomedicine technologies in clinical trials. Since nanomedicine is an interdisciplinary field, a new clinical protocol will need to take into account the multi-dimensional levels of academia, researchers, industry, regulatory agencies, and patients, considering that nanomedicine clinical trials may be significantly different from those for conventional diagnostic and therapeutic agents. The MCDA model will also allow for proper communication channels to ensure a rapid clinical use of safe nanodrugs once they have proceeded and been approved from clinical trials. It is also important to note that the extreme economic pressures faced by researchers and industry experts should not bypass proper clinical protocols and nanomedicine technologies should not be rushed to commercialization without proper risk assessment and risk management.

\section{Afterthoughts}

Nanomedicine offers a wealth of promise and an enthusiastic prospect for breakthroughs in medical diagnosis and treatment. Yet, despite all the exaggerated scenarios of advancing medical technology that will drastically improve human longevity and eradicate all diseases, how concerned should we 
be about the knowledge gaps between research and development that may potentially limit the wonderful applications of nanomedicine technologies? Further, as Raj Bawa and Summer Johnson ask, "will nanomedicine provide valuable contributions to medicine and health care in the long run? It is hard to predict whether nanomedicine will deliver a variety of mostly incremental improvements of existing technologies or whether it will act as a catalyst for a vast technological and healthcare revolution" (Bawa and Johnson 2008, 208).

Advances in technology bend, shift, and transform the limits of what we think is possible. It is virtually impossible to predict the full consequences of all of our actions, and we cannot predict how society and the global order might change in response to new technologies. We may not be able to predict every unprecedented risk from the application of nanotechnology, but science should not ignore our moral or ethical responsibility to consider all the risks either. As Jean-Pierre Dupuy writes, science cannot isolate itself from social responsibility or should be given a monopoly on decision-maker power. Furthermore, "[t]he engineer of tomorrow will not be a sorcerer's apprentice out of negligence or incompetence, but as a matter of method" (Dupuy 2008, xi). Scientists especially need to acknowledge the challenges that might arise with nanotechnology, including the difficulty of implementing a unified code of ethics in a multidisciplinary field, and dealing with public perceptions and (dis)trust. They must also recognize that questions about risk assessment of nanoparticles and nanomaterials in the human body may not always be answered by comparison with conventional pharmaceuticals, and implement stronger regulatory guidelines for evaluating nanodrugs and drug delivery systems, which is difficult due to the level of uncertainty about long-term effects. Clinical trials for nanodrugs and nanomedical technologies must also be carefully evaluated, as they raise other alarming concerns: the unpredictable nature of nanomedicine therapies and technologies makes it difficult for risk assessments, especially for evaluating long-term effects, which in turn complicates informed consent for human participants.

I have argued that understanding epistemic gaps between research and development will allow for a stronger approach for considering any consequences that might arise from the application of nanotechnology, particularly issues of toxicity, the possible complications from nanodrugs and drug delivery systems, and the weak regulatory guidelines governing clinical trials for nanomedicine technologies. I have also argued that the MCDA model might be sufficient for establishing secure communication on multi-dimensional levels, between researchers, stakeholders, and the public. There can be no denying that nanomedicine will transform all segments of society. In order to avoid a public backlash we need to recognize, in the words of K. Eric Drexler, that as the pressures of world competition push us forward, "we need to strike a better balance between our foresight and our rate of advance. We cannot do so 
much to slow the growth of technology, but we can speed the growth of foresight" (Drexler 1990, 203).

\author{
JAIPREET VIRDI \\ IHPST, University of Toronto \\ 91 Charles St. West \\ Toronto, ON \\ Canada M5S $1 \mathrm{~K} 7$ \\ jai.virdi@utoronto.ca
}

\title{
References
}

Allhoff, F. 2008. On the Autonomy and Justification of Nanoethics. In Nanotechnology \& Society: Current and Emerging Ethical Issues, eds. F. Allhoff and P. Lin, 3-38. New York: Springer.

Bawa, R. and S. Johnson. 2007. The Ethical Dimensions of Nanomedicine. In Medical Clinics of North America 881-887.

Bawa, R. and S. Johnson. 2008. Emerging Issues in Nanomedicine and Ethics. In Nanotechnology \& Society: Current and Emerging Ethical Issues, eds. F. Allhoff and P. Lin, 207-233. New York: Springer.

Clement, W. R. 1998. Quantum Jump: A Survival Guide for the New Renaissance. London, Ontario: Insomniac Press.

Dupuy, J. P. 2008. Foreword: The Double Language of Science, and Why It Is So Difficult to Have a Proper Public Debate about the Nanotechnology Program. In Nanotechnology \& Society: Current and Emerging Ethical Issues, eds. F. Allhoff and P. Lin, ix-xiii. New York: Springer.

Drexler, K. E. 1990. Engines of Creation. London: Fourth Estate, Ltd.

Ebbesen, M. and T. G. Jensen. 2006. Nanomedicine: Techniques, Potentials, and Ethical Implications. Journal of Biomedicine and Biotechnology 1-11.

European Group on Ethics in Science and New Technologies, Opinion no.21 (Brussels, January 17, 2007). The full text of the report can be found at http://ec.europa.eu.european group ethics/index en.htm. A condensed version is also available in Allhoff, F. and P. Lin, eds., 2008, Nanotechnology \& Society: Current and Emerging Ethical Issues, New York: Springer, 187-206.

Faunce, T. A. 2007. Nanotherapeutics: New Challenges for Safety and CostEffectiveness Regulation in Australia. Medical Journal of Australia 186(4): 189191.

Fender, J. K. 2008. The FDA and Nano: Big Problems with Tiny Technology. Chicago-Kent Law Review 83.

Freitas Jr., R. A. 2005. What is Nanomedicine? Nanomedicine: Nanotechnology, Biology, and Medicine 1: 2-9. 
Feynman, R. P. 1959. There's Plenty of Room at the Bottom. American Physical Society meeting, Caltech.

Garber, C. 2007. The Potential and the Pitfalls of Nanomedicine. Nanowerk Spotlight.

http://www.nanowerk.com/spotlight/spotid=1891.php.

Keiper, A. 2003. The Nanotechnology Revolution. The New Atlantis Summer 2: 19.

Lazarou, J., B. H. Pomeranz, and P. N. Corey. 1998. Incidence of Adverse Drug Reactions in Hospitalized Patients: A Meta-analysis of Prospective Studies. Journal of the American Medical Association 279(15): 1200-1205.

Lin, P. and F. Allhoff. 2007. Nanoscience and Nanoethics: Defining the Disciplines. In Nanoethics: The Ethical and Social Implications of Nanotechnology, eds. F. Allhoff, P. Lin, J. Moor, and J. Weckert, 3-16. New Jersey: John Wiley \& Sons, Inc.

Lin P., F. Allhoff, J. Moor, and J. Weckert, eds. 2007. Nanoethics: The Ethical and Social Implications of Nanotechnology. New Jersey: John Wiley \& Sons, Inc.

Linkov, I., F. K. Satterstorm and L. M. Corey 2008. Nanotoxicology and Nanomedicine: Making Hard Decisions. Nanomedicine: Nanotechnology, Biology, and Medicine 4(2): 167-171.

Metha, M. D. 2003. The Future of Nanomedicine Looks Promising, But Only if We Learn From the Past. Health Law Review 13(1): 16-18.

Miller, J. 2003. Beyond Biotechnology: FDA Regulation of Nanomedicine. The Columbia Science and Technology Law Review 4: 1-35.

Nanotechnology News. April 22, 2008. FDA Approval for Trials of Nanotechnology Based Cancer Drug Delivery System. http://www.azonano.com/news.asp?newsID=6310.

National Nanotechnology Initiative. 2008. www.nano.gov.

Oberdörser, G., E. Oberdörser and J. Oberdörser. 2007. Concepts of Nanoparticles Dose Metric and Response Metric. Environmental Health Perspectives 115(6): A290.

Preston, C. J. 2006. The Promise and Threat of Nanotechnology: Can Environmental Ethics Guide Us? International Journal for the Philosophy of Chemistry 11(1): 19-44.

Resnik, D. 2006. Clinical Trials of Nanomedicine; Ethical and Safety Issues. Research Practitioner.

http://www.accessmylibrary.com/coms2/summary 0286-29032454 ITM.

Roco M. C. 2007. Foreword: Ethical Choices in Nanotechnology Development. In Nanoethics: The Ethical and Social Implications of Nanotechnology, eds. F. Allhoff, P. Lin, J. Moor, and J. Weckert, xi-xiii. New Jersey: John Wiley \& Sons, Inc. 
Rosenberg, W. and A. Donald. 1995. Education and Debate: Evidence Based Medicine: An Approach to Clinical Problem-solving. British Medical Journal 310: 1122-1126.

Shew, A. 2008. Nanotechnology's Future: Considerations for the Professional. In Nanotechnology \& Society: Current and Emerging Ethical Issues, eds. F. Allhoff and P. Lin, 127-146. New York: Springer.

Stolberg, S. G. November 28, 1999. The Biotech Death of Jesse Gelsinger. The New York Times Magazine Online. http://query.nytimes.com/gst/fullpage.html?res=9C03E4DE1F3CF93BA15752 C1A96F958260.

Thompson, P. B. 2008. The Presumptive Case for Nanotechnology. In Nanotechnology \& Society: Current and Emerging Ethical Issues, eds. F. Allhoff and P. Lin, 39-54. New York: Springer.

Till, M. C., M. M. Simkin, and S. Maebius. 2005. Nanotech Meets the FDA: A Success Story about the First Nanoparticulate Drugs Approved by the FDA. Nanotechnology Law and Business 2(2): 163-167.

SCENIHR Report. Sept. 2005. The Synthesis Report on the Public Consultation of the SCENIHR Opinion on 'The Appropriateness of Existing Methodologies to Assess the Potential Risks Associated with Engineered and Adventitious Products of Nanotechnologies.'

http://ec.europa.eu/health/ph risk/documents/synth report.pdf.

Upshur, R. E. G. and C. S. Tracy. 2004. Legitimacy, Authority, and Hierarchy: Critical Challenges for Evidence-Based Medicine. Brief Treatment and Crisis Intervention 4: 197-204.

Van den Hoven, J. 2008. The Tangled Web of Tiny Things: Privacy Implications of Nano-electronics. In Nanotechnology \& Society: Current and Emerging Ethical Issues, eds. F. Allhoff and P. Lin, 147-162. New York: Springer.

Wagner, V. A. Dullaart, A. K. Bock, and A. Zweck. 2006. The Emerging Nanomedicine Landscape. Nature Biotechnology 24(10): 1214. 